\title{
Using the Bootstrap to Account for Linkage Errors when Analysing Probabilistically Linked Categorical Data
}

\author{
James O. Chipperfield ${ }^{1}$ and Raymond L. Chambers ${ }^{2}$
}

\begin{abstract}
Record linkage is the act of bringing together records that are believed to belong to the same unit (e.g., person or business) from two or more files. Record linkage is not an error-free process and can lead to linking a pair of records that do not belong to the same unit. This occurs because linking fields on the files, which ideally would uniquely identify each unit, are often imperfect. There has been an explosion of record linkage applications, particularly involving government agencies and in the field of health, yet there has been little work on making correct inference using such linked files. Naively treating a linked file as if it were linked without errors can lead to biased inferences. This article develops a method of making inferences for cross tabulated variables when record linkage is not an error-free process. In particular, it develops a parametric bootstrap approach to estimation which can accommodate the sophisticated probabilistic record linkage techniques that are widely used in practice (e.g., 1-1 linkage). The article demonstrates the effectiveness of this method in a simulation and in a real application.
\end{abstract}

Key words: Record linkage; measurement error; parametric bootstrap.

\section{Introduction}

Record linkage is the act of bringing together records from two or more files that are believed to belong to the same unit in a defined population (e.g., a person or business). Record linkage is an appropriate technique when these data sets are joined to enhance dimensions such as time and breadth or depth of detail. In particular, record linkage is an intrinsic part of virtually all coverage error estimation and correction methodologies, where records from two or more frames, each with incomplete coverage of a target population, are linked in order to estimate the extent of the overlap of these frames. In such cases, coverage error models are usually based on the linked data. Ideally, the linkage will be perfect, that is, all records belonging to the same unit are linked and there are no links between records that belong to different units. However, in many situations perfect linkage is not possible. This is because linking fields (e.g., name, address, postcode) may not uniquely identify a unit, legitimately change over time, be missing or contain errors.

\footnotetext{
1 Australian Bureau of Statistics, Methodology Division, P O Box 10, Belconnen, Australian Capital Territory 2616 Australia. Email: james.chipperfield@abs.gov.au

${ }^{2}$ University of Wollongong, National Institute for Applied Statistics Research, Northfields Avenue Wollongong, New South Wales, 2500 Australia. Email: ray@uow.edu.au

Acknowledgments: We would like to thank the referees for their attention to detail and expertise. Their efforts significantly improved this article.
} 
Probabilistic record linkage is a widely used approach to record linkage. In probabilistic record linkage (Fellegi and Sunter 1969) each possible link, called a record pair, is given a score based on the likelihood that the two records belong to the same unit. Optimisation algorithms are then used to select which record pairs are declared as links. Probabilistic methods for record linkage are now well established (see Herzog et al. 2007; Winkler 2001; Winkler 2005) and there is a range of computer packages available to implement them. A recent example of probabilistic linkage from the Australian Bureau of Statistics (Zhang and Campbell 2012) is the linkage of person records on its 2006 and 2011 Censuses of Population and Housing to facilitate analysis of how characteristics of cohorts change over time. There are many other examples of probabilistic record linkage from statistical agencies, particularly in the area of health data (see the introduction of Kim and Chambers 2012a).

Naively treating a probabilistically linked file as if it is perfectly linked leads to biased inference. Scheuren and Winkler (1993) and Lahiri and Larsen (2005) (referred to as SW and LL hereafter) propose bias-corrected estimators of coefficients in a linear regression model given data from a probabilistically linked file. Chipperfield et al. (2011) consider the analysis of linked binary variables. Building on Chambers (2009), Kim and Chambers (2012a, 2012b) (referred to as KC hereafter) investigate the analysis of linked data using a more general set of models fitted using estimating equations. Kim and Chambers (2012b) review recent development in inference for regression parameters using linked data.

Linkage models form the key feature of all of the above approaches. The linkage model describes the probability that a record on one file is linked to each of the records on another file. For a linkage model to be useful, it must properly take into account how records were linked. SW and LL do not allow for 1-1 linkage, where every record on one file is linked to a distinct and different record on the other, or for linkage in multiple passes or stages, both of which are commonly used in probabilistic record linkage. In theory, KC allows for 1-1 linkage, but imposes strong constraints on the linkage model in order to do so. $\mathrm{KC}$ also requires a clerical sample to estimate the parameters of the linkage model, something which is not always available in practice and which itself can be subject to measurement errors.

This article describes an approach to inference using estimating equations that is based on probabilistically linked data where the linked data file is created under the 1-1 constraint. In fact, the proposed method is valid when the linkage is performed in an arbitrary fashion, as long as the linkage process itself is probabilistic and can be replicated. In particular, we argue that replication is straightforward within the probabilistic record linkage framework of Fellegi and Sunter (1969).

Section 2 introduces the basics of record linkage and the linkage model. It describes a bootstrap approach to fitting the linkage model and compares it with the approach of LL. Section 3 describes how this approach to linkage error modelling can be used to bias correct cross tabulations based on linked data, as well as to make correct inference for binary variables. Section 4 demonstrates through simulation that the proposed approach has good bias and coverage properties. Section 5 considers the performance of the proposed approach for estimating regression coefficients in a real example. Section 6 contains some concluding remarks. 


\section{Linkage and Linkage Models}

This section introduces the basics of record linkage. It also defines the linkage error model, which is an essential ingredient for making correct inference with probabilistically linked data, and proposes a bootstrap approach to estimating it. One crucial aspect of our model is that it distinguishes between the process of linking and whether or not the linking is successful. Here, linking denotes the putting together of records to make up the linked data file, that is, the identification of records that are believed to come from the same population unit, while successful linking denotes the event that two records actually come from the same population unit.

\subsection{A Framework for the Probability Linking of Two Files of the Same Size}

Consider linking two files, file $X$ and file $Y$, each containing $N$ records that correspond to the same set of units. Let $i=1, \ldots, N$ denote an arbitrary indexing of the records in $X$, and similarly let $k=1, \ldots, N$ denote an arbitrary indexing of the records in $Y$. A subset of the set of pairs $(i, k)$ is chosen to define the linked records, and we refer to this subset as the set of linked pairs. In addition, let $j=1, \ldots, N$ denote another indexing of the records on $Y$ such that record $i$ in $X$ and record $j$ in $Y$ is a correct link when $i=j$. It is important to note that the $j$ index of a record in $Y$ is unknown, since it by definition requires knowledge of the correctly linked data file.

Suppose that there are $L$ linking fields defined by variables that are common to $X$ and $Y$. We then define $\mathbf{A}^{o}=\left(\mathbf{A}_{11}^{o}, \ldots, \mathbf{A}_{i k}^{o}, \ldots, \mathbf{A}_{N N}^{o}\right)$ to be the $L \times N^{2}$ matrix of observed agreement patterns for all record pairs, that is $\left(\mathbf{A}_{i k}^{o}\right)^{T}=\left(A_{i k 1}^{o}, A_{i k l}^{o}, \ldots, A_{i k L}^{o}\right)$, where $A_{i k l}^{o}=0$ or 1 if the linked $(i, k)$ pair disagrees or agrees on the $l$ th linking field, respectively. For example, if first name, last name and date-of-birth are the three linking fields, and if the $(i, k)$ th pair agrees on the first two but not on the third, then $\left(\mathbf{A}_{i k}^{o}\right)^{T}=\left(\begin{array}{lll}1 & 1 & 0\end{array}\right)$. Here ' 1 ' indicates agreement and ' 0 ' indicates disagreement. There are $2^{L}$ possible agreement patterns for a record pair.

Define $\mathbf{A}=\left(\mathbf{A}_{11}, \ldots, \mathbf{A}_{i j}, \ldots, \mathbf{A}_{N N}\right)$ to be the matrix of unobserved agreement patterns, where $\mathbf{A}_{i j}^{T}=\left(A_{i j 1}, A_{i j l}, \ldots, A_{i j L}\right)$, with $A_{i j l}=0$ or 1 if the $(i, j)$ th record pair disagrees or agrees on the $l$ th linking field, respectively. Although $\mathbf{A}$ is simply a rearrangement of the columns of $\mathbf{A}^{o}$, this rearrangement is indexed by the unobserved $j$ index. $\mathbf{A}$ is therefore a latent variable and can be modelled as the outcome of a random process. A common model for $\mathbf{A}$, and one which we use in this article, is often described by the following set of parameters:

- $M_{i i l}=\operatorname{Pr}\left(A_{i i l}=1 \mid i=j\right)$ the probability that the value of the $l$ th linking field for record $i$ in $X$ is the same as the corresponding value for linked record $i$ in $Y$;

- $U_{i j l}=\operatorname{Pr}\left(A_{i j l}=1 \mid i \neq j\right)$ : the probability that the values of the $l$ th linking field for record $i$ in $X$ and record $j$ in $Y$ are the same, given $i \neq j$.

The probabilities $M_{i l}$ and $U_{i j l}$ are often assumed to be homogeneous, that is, they do not depend on $i$ and $j$. In such a situation we denote them by $M_{l}$ and $U_{l}$, respectively. Conditional independence is often also assumed. Conditional independence means that for any linked pair, agreement on linking field $l$ is independent of agreement on any other 
linking field $l^{\prime}$ for all values $l^{\prime} \neq l$. This is a strong assumption but, as we will see, it can be a reasonable working assumption.

Put $\boldsymbol{\psi}=\left(M_{1}, \ldots, M_{L}, U_{1}, \ldots, U_{L}\right)$. Prior to probabilistic linking, $\boldsymbol{\psi}$ or its estimate, $\hat{\boldsymbol{\psi}}$, is required. In some cases $\boldsymbol{\psi}$ may be known (e.g., if a unique identifier was available from a previous linkage of $X$ and $Y$ ). Computing $\hat{\psi}$ using mixture models has been extensively studied (see Larsen and Rubin 2001, who also consider relaxing the conditional independence assumption).

\subsection{The Linkage Process}

Given a value for $\boldsymbol{\psi}$, and a proposed indexing of $Y$ defined by $k=1, \ldots, N$, Fellegi and Sunter (1969) suggested calculating a weight for the observed $(i, k)$ th pair of the form $W_{i k}^{o}=\sum_{l} w_{i k l}^{o}$, where

$$
\begin{aligned}
w_{i k l}^{o} & =\ln \left(M_{l} / U_{l}\right) & & \text { if } A_{i k l}^{o}=1 \\
& =\ln \left[\left(1-M_{l}\right) /\left(1-U_{l}\right)\right] & & \text { if } A_{i k l}^{o}=0 .
\end{aligned}
$$

These authors argue that the larger this pair weight, the more likely that the pair is a correct link. These pair weights are then used in an optimisation algorithm to determine the set of $(i, k)$ pairs that are declared as links. An obvious objective function to maximise is $O=\sum_{i, k} W_{i k}^{o} L_{i k}$, where $L_{i k}=1$ if the $(i, k)$ pair is linked and $\sum_{k} L_{i k}=1$ for all $i$ and $k$. Often a 1-1 constraint is imposed such that $\sum_{k} L_{i k}=\sum_{i} L_{i k}=1$ for all $i$ and $k$. Also, in practice a linked pair must have a weight that is greater than a cut-off value, $c_{o}$, to be declared a link. The value for $c_{o}$ can be chosen to ensure that the proportion of links that are correct is acceptably high (see Herzog et al. 2007).

To keep computations to a practical level, records on $X$ and $Y$ are often assigned to blocks, where only records within the same block form linked pairs. If there is more than one suitable blocking field, linking can often be performed in multiple passes, where a different set of blocking and linking fields is used in each pass. For example, Chipperfield et al. (2011) consider an example with two passes.

\subsection{The Linkage Model}

The result of a 1-1 linkage process is a generally unknown permutation matrix $\mathbf{P}=\left[\delta_{i j}\right]$ with $(i, j)$ element $\delta_{i j}$ equal to 1 if record $i$ in $X$ is linked to record $j$ in $Y$ and equal to 0 otherwise. By definition of the $i$ and $j$ indices, diagonal entries of 1 on $\mathbf{P}$ indicate correct links. Let $\mathbf{V}^{(X)}$ denote a matrix of values derived from the information in $X$. We then put

$$
E\left(\mathbf{P} \mid \mathbf{V}^{(X)}\right)=\mathbf{Q}
$$

We refer to (1) as the linkage model. Specifically, the linkage model is given by $\mathbf{Q}=\left[q_{i j}\right]$ where $q_{i j}=E\left(\delta_{i j} \mid \mathbf{V}^{(X)}\right)$ is the probability that record $i$ in $X$ is linked to record $j$ in $Y$, so $q_{i i}$ is the probability of correctly linking to record $i$ in $X$, and $\sum_{j} q_{i j}=\sum_{i} q_{i j}=1$. Various authors estimate $\mathbf{Q}$ in different ways. 
Let $I_{i j}$ denote the indicator for $i=j$. Chambers (2009) considers an 'exchangeable' linkage model, where

$$
q_{i j}=\lambda \mathrm{I}_{i j}+(1-\lambda)(N-1)^{-1}\left(1-I_{i j}\right)
$$

This model is constrained through a single parameter, $\lambda$ (or one parameter per block, if a blocking strategy is used). In practice, $\lambda$ is unknown; Chambers (2009) suggests that in such a situation, it can be estimated using a sample of linked pairs that are reviewed clerically and are assigned, without error, as matches (correct links) or nonmatches (incorrect links). However, note that (2) does not explicitly account for how records are linked (e.g., single pass vs. multiple passes), and so may be inadequate when the method of linking leads to heterogeneous correct (and incorrect) linkage probabilities.

LL on the other hand implicitly assume that ordering $Y$ by the $j$ and $k$ indices leads to the same result, and so put $\mathbf{Q}=\left[q_{i k}\right]$, where $q_{i k}$ is estimated by $q_{i k}^{(L L)}=p_{i k} / \sum_{k^{\prime}} p_{i k^{\prime}}$, where $p_{i k}$ is the probability that the (i,k) pair is a correct link under the model for $\mathbf{A}$ in Subsection 2.1 (see LL, page 223 for an expression for $p_{i k}$ based on a mixture model). By definition, $q_{i k}$ is then the probability that the $(i, k)$ pair is linked. Since the probability of linkage of two records is not the same as the probability that these two records, when linked, are correctly linked, the use of $q_{i k}$ as a proxy for $q_{i j}$ is incorrect in general and, as we show later, can lead to significant bias. Moreover, the estimator $q_{i k}^{(L L)}$ does not factor in all of the complexities of the linking process (e.g., 1-1 linkage), with LL (page 226) noting that "It is not entirely clear how to force one-to-one matches and consider probabilities of matching in which two records in one file have a nonzero probability of matching a record in the second file." Goldstein et al. (2012) make a similar proxy assumption, suggesting the estimator $q_{i k}^{(G S)}=W_{i k}^{o} / \sum_{k^{\prime}} W_{i k^{\prime}}^{o}$. In the following section, we define a bootstrap approach to estimating $\mathbf{Q}$ for a linkage process which may include 1-1 assignment.

\subsection{A Bootstrap Estimator of $Q$}

We assume that the linking process can be characterised as in Subsection 2.2 and that all linking fields on $X$ and $Y$ are known. We also assume that the conditional independence model (see Subsection 2.1) holds and that an unbiased estimator, denoted by $\hat{\boldsymbol{\psi}}$, of the vector of $M$ and $U$ probabilities defined there is available. Note that if either of these assumptions does not hold, then the bootstrap estimator of $\mathbf{Q}$ defined below may well be biased. In particular, following Winglee et al. (2005), we estimate $\mathbf{Q}$ by bootstrap replication of the linking process. This is accomplished by bootstrapping the unobserved agreement pattern matrix, $\mathbf{A}$, assuming that patterns defined by distinct pairs of population units are independently distributed. That is, for each bootstrap realisation of the linking process, we simulate $N^{2}$ realisations $\mathbf{A}_{i j}^{*}=\left(A_{i j l}^{*}\right)$ of $\mathbf{A}_{i j}$ such that

$$
A_{i j l}^{*}= \begin{cases}B\left(\hat{M}_{l}\right) & i=j \\ B\left(\hat{U}_{l}\right) & i \neq j\end{cases}
$$

where $B(\pi)$ denotes an independent realisation of a Bernoulli random variable with success probability $\pi$. Note that since bootstrap replication aims to generate agreement patterns that have a similar distribution to the actual unobserved set of agreement 
patterns $\mathbf{A}$, this assumption of independent Bernoulli realisations is a strong one. Alternative models for $\mathbf{A}$ can be constructed, using the fact that this matrix defines a network connecting the population units. Further research is required on whether this extra level of sophistication is warranted, however.

Given this bootstrap realisation of $\mathbf{A}$, we obtain the corresponding bootstrap realisation of the linkage matrix $\mathbf{P}$ by repeating the linking process using the bootstrap weights $W_{i j}^{*}=\sum_{l} w_{i j l}^{*}$, where

$$
\begin{aligned}
w_{i j l}^{*} & =\ln \left(\hat{M}_{l} / \hat{U}_{l}\right) & & \text { if } A_{i j l}^{*}=1 \\
& =\ln \left[\left(1-\hat{M}_{l}\right)\left(1-\hat{U}_{l}\right)\right] & & \text { if } A_{i j l}^{*}=0 .
\end{aligned}
$$

We then estimate $\mathbf{Q}$ by averaging over these bootstrap realisations of $\mathbf{P}$. That is, we proceed as follows:

1. Repeat the following steps a total of $B$ times:

a. Generate $\mathbf{A}(b)$ as the $b$ th independent draw of $\mathbf{A}$ based on $\hat{\boldsymbol{\psi}}$ and an assumption of independent Bernoulli realisations.

b. Calculate the linking weights, $W_{i j}^{(b)}$ for all $i, j$ and $l$, as a function of $\hat{\boldsymbol{\psi}}$ and $\mathbf{A}(b)$.

c. Link $X$ and $Y$ using the $W_{i j}^{(b)}$ using the same algorithm that was used to link the original file. Denote the resulting $N \times N$ permutation matrix of actual links by $\mathbf{P}(b ; \hat{\boldsymbol{\psi}})$, that is the columns of $\mathbf{P}$ are indexed by $j$ and element $(i, j)$ of $\mathbf{P}$ is equal to 1 if record $i$ in $X$ is linked to record $j$ in $Y$ and 0 otherwise. Note that true links then correspond to record pairs where the $(i, i)$ element of $\mathbf{P}$ is equal to 1 .

2. Estimate $\mathbf{Q}$ by $\hat{\mathbf{Q}}(\hat{\boldsymbol{\psi}})=B^{-1} \sum_{b} \mathbf{P}(b ; \hat{\boldsymbol{\psi}})$.

Note that if $X$ and $Y$ are 1-1 linked, then they must also be linked in this fashion in Step 1(c) above.

\section{Estimation of Frequency Tables from Linked Data}

\subsection{A Bias-Corrected Estimator}

Let $y$ be a categorical variable recorded on file $Y$ with categories $y=1, \ldots, u, \ldots, Y$, and let $x$ be a categorical variable recorded on file $X$ with categories $x=1, \ldots, t, \ldots, T$. The values of $x$ and $y$ for the correct links are denoted by $x_{i}$ and $y_{i}$, respectively. Given $X$ we can then define the $N \times T$ incidence matrix $\mathbf{I}^{(X)}=\left[I_{i t}^{(X)}\right]$, where $I_{i t}^{(X)}$ is the indicator for $x_{i}=t$. Similarly, given $Y$ we can define the $N \times Y$ incidence matrix $\mathbf{I}^{(Y)}=\left[I_{i u}^{(Y)}\right]$, where $I_{i u}^{(Y)}$ is the indicator for $y_{i}=u$. The $T \times Y$ matrix of frequencies of interest is $\mathbf{N}=\left(\mathbf{I}^{(X)}\right)^{T} \mathbf{I}^{(Y)}$ where the $(t, u)$ element of $\mathbf{N}$ is $N_{t u}$.

Assuming independent and identically distributed population units, the probability distribution for $(x, y)$ is multinomial with parameter $\pi=\left[\pi_{t u}\right]$, where $\pi_{t u}$ is the probability that $(x, y)=(t, u)$. Under the multinomial model, $E\left(\mathbf{I}^{(Y)} \mid \mathbf{I}^{(X)}\right)=\Delta$ where $\Delta=\Delta(\pi)$ has $(i, u)$ element,

$$
E\left(I_{i u}^{(Y)} \mid I_{i t}^{(X)}\right)=\pi_{u \mid t} I_{i t}^{(X)}
$$


with $\pi_{u \mid t}=\pi_{t u}\left(\sum_{u^{\prime}} \pi_{t u^{\prime}}\right)^{-1}$. Let $y_{i}^{*}$ denote the linked value of $y$ for record $i$ in $X$. The naive estimator of $\mathbf{N}$, which is based on the assumption that all links are correct, is then

$$
\mathbf{N}^{*}=\left(\mathbf{I}^{(X)}\right)^{T} \mathbf{I}^{\left(Y^{*}\right)}=\left(\mathbf{I}^{(X)}\right)^{T} \mathbf{P} \mathbf{I}^{(Y)},
$$

where $\mathbf{I}^{\left(Y^{*}\right)}=\left[I_{i u}^{\left(Y^{*}\right)}\right]$, and $I_{i u}^{\left(Y^{*}\right)}$ is the indicator for $y_{i}^{*}=u$.

A key assumption we now make is that of conditional independence of the population distribution of $y$ and the linkage error matrix $\mathbf{P}$ given the values in $X$. This allows us to write

$$
E\left(\mathbf{P} \mathbf{I}^{(Y)} \mid \mathbf{I}^{(X)}\right)=E\left(\left(\mathbf{P} \mid \mathbf{I}^{(X)}\right)\right) E\left(\mathbf{I}^{(Y)} \mid \mathbf{I}^{(X)}\right) .
$$

Given this assumption, the naive estimator $\mathbf{N}^{*}$ is a biased predictor of $\mathbf{N}$, since

$$
\begin{aligned}
& E\left(\mathbf{N}^{*}-\mathbf{N} \mid \mathbf{I}^{(X)}\right)=\left(\mathbf{I}^{(X)}\right)^{T}\left\{E\left(\mathbf{P} \mid \mathbf{I}^{(X)}\right) E\left(\mathbf{I}^{(Y)} \mid \mathbf{I}^{(X)}\right)-E\left(\mathbf{I}^{(Y)} \mid \mathbf{I}^{(X)}\right)\right\} \\
& =\left(\mathbf{I}^{(X)}\right)^{T}\left(\mathbf{Q}-\mathbf{I}_{N}\right) \Delta(\pi)
\end{aligned}
$$

which is nonzero in general. Here $\mathbf{I}_{N}$ is the identity matrix of order $N$. Using $\hat{\mathbf{Q}}=\hat{\mathbf{Q}}(\hat{\boldsymbol{\psi}})$ as an estimate of $\mathbf{Q}=\mathbf{Q}(\boldsymbol{\psi})$ leads to the bias-corrected estimator

$$
\hat{\mathbf{N}}^{*}=\left(\mathbf{I}^{(X)}\right)^{T}\left\{\mathbf{I}^{\left(Y^{*}\right)}-\left(\hat{\mathbf{Q}}-\mathbf{I}_{N}\right) \Delta(\hat{\pi})\right\}
$$

where $\hat{\boldsymbol{\pi}}$ is an estimate of $\boldsymbol{\pi}$ defined by

1. Initialising $\hat{\boldsymbol{\pi}}$ by $\hat{\boldsymbol{\pi}}^{(0)}$.

2. Computing $\hat{\mathbf{N}}^{*(h)}=\left[\hat{N}_{u t}^{*(h)}\right]=\hat{\mathbf{N}}^{*}\left(\hat{\boldsymbol{\pi}}^{(h)}\right)$.

3. Computing $\hat{\boldsymbol{\pi}}^{(h+1)}$ using $\hat{\pi}_{t u}^{(h+1)}=\max \left(\hat{N}_{u t}^{*(h)} N^{-1}, 0\right)$.

4. Iterating between Steps 2 and 3 above until convergence.

In all applications reported later in this article, $\hat{\boldsymbol{\pi}}^{(0)}$ was based on the naive estimate $\mathbf{N}^{*}$ and this iterative scheme always converged.

\subsection{Variance Estimation}

Let $\hat{N}_{t u}^{*}(\hat{\psi})$ denote the $(t, u)$ element of $\hat{\mathbf{N}}^{*}$ given by (4), and put $\mathbf{I}_{t}^{(X)}=\left(I_{t u}^{(X)}, 1 \leq u \leq Y\right)$, $\mathbf{I}_{u}^{(Y)}=\left(I_{t u}^{(Y)}, 1 \leq t \leq T\right)$ and $\mathbf{I}_{u}^{\left(Y^{*}\right)}=\left(I_{t u}^{\left(Y^{*}\right)}, 1 \leq t \leq T\right)$. We can write

$$
\begin{aligned}
\operatorname{Var}\left(\hat{N}_{t u}^{*}(\hat{\psi})\right) & =\operatorname{Var}\left\{E\left(\hat{N}_{t u}^{*}(\hat{\psi}) \mid \mathbf{I}_{t}^{(X)}, \mathbf{I}_{u}^{\left(Y^{*}\right)}, \mathbf{I}_{u}^{(Y)} ; \psi, \pi\right)\right\}+E\left\{\operatorname{Var}\left(\hat{N}_{t u}^{*}(\hat{\psi}) \mid \mathbf{I}_{u}^{(Y)}, \mathbf{I}_{u}^{\left(Y^{*}\right)}, \mathbf{I}_{t}^{(X)} ; \psi, \pi\right)\right\} \\
& =\operatorname{Var}\left\{E\left(\hat{N}_{t u}^{*}(\hat{\psi}) \mid \mathbf{I}_{t}^{(X)}, \mathbf{I}_{u}^{\left(Y^{*}\right)}, \mathbf{I}_{u}^{(Y)} ; \psi, \pi\right)\right\}+V_{t u}^{(\hat{\psi})}
\end{aligned}
$$

where we identify $V_{t u}^{(\hat{\psi})}$ as the component of variance due to estimation of $\boldsymbol{\psi}$. We then make the large sample approximation

$$
\operatorname{Var}\left\{E\left(\hat{N}_{t u}^{*}(\hat{\psi}) \mid \mathbf{I}_{t}^{(X)}, \mathbf{I}_{u}^{\left(Y^{*}\right)}, \mathbf{I}_{u}^{(Y)} ; \psi, \pi\right)\right\} \approx \operatorname{Var}\left(\hat{N}_{t u}^{*}(\psi) ; \psi, \pi\right)
$$

Proceeding along the same lines, and using the conditional independence assumption, 
we have

$$
\begin{aligned}
& \operatorname{Var}\left(\hat{N}_{t u}^{*}(\boldsymbol{\psi}) ; \boldsymbol{\psi}, \boldsymbol{\pi}\right)=\operatorname{Var}\left\{E\left(\hat{N}_{t u}^{*}(\psi) \mid \mathbf{I}_{t}^{(X)}, \mathbf{I}_{u}^{(Y)} ; \psi\right) ; \pi\right\}+E\left\{\operatorname{Var}\left(\hat{N}_{t u}^{*}(\psi) \mid \mathbf{I}_{t}^{(X)}, \mathbf{I}_{u}^{(Y)} ; \psi\right) ; \pi\right\} \\
& =V_{t u}^{(y)}+E\left\{\operatorname{Var}\left(\hat{N}_{t u}^{*}(\psi) \mid \mathbf{I}_{t}^{(X)}, \mathbf{I}_{u}^{(Y)} ; \psi\right) ; \pi\right\} \\
& =V_{t u}^{(y)}+E\left\{\operatorname{Var}\left(\hat{N}_{t u}^{*}(\psi) \mid \mathbf{I}_{t}^{(X)} ; \psi\right) ; \pi\right\} \\
& =V_{t u}^{(y)}+V_{t u}^{*}
\end{aligned}
$$

where $V_{t u}^{(y)}$ and $V_{t u}^{*}$ now denote the components of variance, due to the multinomial model and the linkage errors respectively. That is, we write down the large sample approximation

$$
\operatorname{Var}\left(\hat{N}_{t u}^{*}(\hat{\psi})\right) \approx V_{t u}^{(y)}+V_{t u}^{*}+V_{t u}^{(\hat{\psi})}
$$

Note that since $\mathbf{Q}$ is determined by $\psi, V_{t u}^{(\hat{\psi})}$ can also be considered to be the component of variance due to estimating $\mathbf{Q}$.

In order to estimate (5), we start by writing down a large sample approximation to $V_{t u}^{(y)}$ of the form

$$
\begin{aligned}
V_{t u}^{(y)} & =\operatorname{Var}\left[E\left\{\hat{\mathbf{N}}_{t u}^{*}(\psi) \mid \mathbf{I}_{t}^{(X)}, \mathbf{I}_{u}^{(Y)} ; \psi\right\} ; \pi\right] \\
& =\operatorname{Var}\left\{E\left(\left(\mathbf{I}_{t}^{(X)}\right)^{T} \mathbf{I}_{u}^{\left(Y^{*}\right)}-\left[\left(\mathbf{I}_{t}^{(X)}\right)^{T}\left(\mathbf{Q}-\mathbf{I}_{N}\right) \Delta(\hat{\pi})\right]_{t u} \mid \mathbf{I}_{t}^{(X)} ; \psi\right) ; \pi\right\} \\
& \approx \operatorname{Var}\left\{E\left(\left(\mathbf{I}_{t}^{(X)}\right)^{T} \mathbf{I}_{u}^{\left(Y^{*}\right)}-\left[\left(\mathbf{I}_{t}^{(X)}\right)^{T}\left(\mathbf{Q}-\mathbf{I}_{N}\right) \Delta(\pi)\right]_{t u} \mid \mathbf{I}_{t}^{(X)} ; \psi\right) ; \pi\right\} \\
& =\operatorname{Var}\left(\left(\mathbf{I}_{t}^{(X)}\right)^{T} \mathbf{Q} \mathbf{I}_{u}^{(Y)} \mid \mathbf{I}_{t}^{(X)} ; \pi\right) \\
& =\left(\mathbf{I}_{t}^{(X)}\right)^{T} \mathbf{Q} \operatorname{Var}\left(\mathbf{I}_{u}^{(Y)} \mid \mathbf{I}_{t}^{(X)} ; \pi\right) \mathbf{Q}^{T} \mathbf{I}_{t}^{(X)}
\end{aligned}
$$

This suggests the plug-in estimator

$$
\hat{V}_{t u}^{(y)}=\left(\mathbf{I}_{t}^{(X)}\right)^{T} \hat{\mathbf{Q}} \hat{V}\left(\mathbf{I}_{u}^{(Y)} \mid \mathbf{I}_{t}^{(X)} ; \hat{\pi}\right) \hat{\mathbf{Q}}^{T} \mathbf{I}_{t}^{(X)}
$$

where $\hat{V}\left(\mathbf{I}_{u}^{(Y)} \mid \mathbf{I}_{t}^{(X)} ; \hat{\boldsymbol{\pi}}\right)$ is a diagonal matrix with $i$ th diagonal element $\left(1-\hat{\pi}_{u \mid t}\right) \hat{\pi}_{u \mid t}$.

We estimate $V_{t u}^{*}$ by parametric bootstrapping (Lahiri 2003). That is, given $x_{i}=t$, we first generate $\mathrm{S}$ independent values $y_{i s}$, for $s=1, \ldots, S$, of $y_{i}$ by making random draws from the multinomial distribution with parameter $\hat{\boldsymbol{\pi}}_{\mid t}=\left(\hat{\pi}_{u \mid t}\right)$, setting $I_{i u}^{(Y)}(s)$ equal to the indicator for $y_{i s}=u$. If we write the corresponding simulated true incidence matrix as $\mathbf{I}^{(Y)}(s)=\left[I_{i u}^{(Y)}(s)\right]$, the $b$ th bootstrap value for the simulated linked incidence matrix $\mathbf{I}^{\left(Y^{*}\right)}(s)$ is $\mathbf{I}^{\left(Y^{*}\right)}(b, s)=\mathbf{P}(b) \mathbf{I}^{(Y)}(s)$, where $\boldsymbol{P}(b)$ is the $b$ th bootstrap value of $\mathbf{P}$, obtained using the procedure described in Subsection 2.4. Our estimate $V_{t u}^{*}$ is then

$$
\hat{V}_{t u}^{*}=S^{-1} \sum_{s} B^{-1} \sum_{b}\left(\hat{N}_{t u}^{*}(b, s)-N_{t u}(s)\right)^{2},
$$

where $\hat{N}_{t u}^{*}(b, s)$ is the $(t, u)$ element of $\hat{\mathbf{N}}^{*}(b, s)=\left(\mathbf{I}^{(X)}\right)^{T}\left\{\mathbf{I}^{\left(Y^{*}\right)}(b, s)-\left(\hat{\mathbf{Q}}-\mathbf{I}_{N}\right) \Delta(\hat{\pi})\right\}$ and $N_{t u}(s)$ is the corresponding element of $\hat{\mathbf{N}}^{*}(s)=\left(\mathbf{I}^{(X)}\right)^{T}\left\{\mathbf{I}^{\left(Y^{*}\right)}(s)-\left(\hat{\mathbf{Q}}-\mathbf{I}_{N}\right) \Delta(\hat{\pi})\right\}$. 
Finally, we use a double bootstrap version of the procedure described in Subsection 2.4 to derive an independent bootstrap estimator for $V_{t u}^{(\hat{\psi})}$. Let $s=1, \ldots, S$ index the multinomial model-based parametric simulations of $y$ described in the preceding paragraphs. Indexing these new double bootstrap replications by $r=1, \ldots, R$, we proceed as follows:

1. Generate $\mathbf{A}(r)$ so that $\operatorname{Pr}\left(A_{i i l}(r)=1\right)=\hat{M}_{l}$ and $\operatorname{Pr}\left(A_{i j l}(r)=1\right)=\hat{U}_{l}$;

2. Calculate $\hat{\psi}(r)$ (and hence $\hat{M}_{l}(r)$ and $\hat{U}_{l}(r)$ ) from $\mathbf{A}(r)$ in the same way that $\hat{\psi}$ was calculated from $\mathbf{A}$;

3. Calculate $\hat{\mathbf{Q}}(r)$ from $\hat{\psi}(r)$ in the same way that $\hat{\mathbf{Q}}$ was calculated from $\hat{\boldsymbol{\psi}}$. Specifically, this involves for $b=1, \ldots, B$,

a. Generating $\mathbf{A}(r, b)$ so that $\operatorname{Pr}\left(A_{i i l}(r, b)=1\right)=\hat{M}_{l}(r)$ and $\operatorname{Pr}\left(A_{i j l}(r, b)=1\right)=$ $\hat{U}_{l}(r)$;

b. Generating $\mathbf{P}(r, b)$ as a random realisation of the permutation matrix that characterises probabilistic linkage with agreement patterns $\mathbf{A}(r, b)$ and $\psi=\hat{\psi}(r)$

4. Calculate $\hat{\mathbf{Q}}(r)=B^{-1} \sum_{b} \mathbf{P}(r, b)$ and hence $\hat{\mathbf{N}}^{*}(r, s)=\left[\hat{N}_{t u}^{*}(r, s)\right]$, where $\hat{\mathbf{N}}^{*}(r, s)=\left(\mathbf{I}^{(X)}\right)^{T}\left\{\mathbf{I}^{\left(Y^{*}\right)}(s)-\left(\hat{\mathbf{Q}}(r)-\mathbf{I}_{N}\right) \Delta(\hat{\pi})\right\}$.

Our bootstrap estimate of $V_{t u}^{(\hat{\psi})}$ is then

$$
\hat{V}_{t u}^{(\hat{\psi})}=S^{-1} \sum_{s} R^{-1} \sum_{r}\left(\hat{N}_{t u}^{*}(r, s) \underset{1 \leq r^{\prime} \leq R}{a v}\left\{\hat{N}_{t u}^{*}\left(r^{\prime}, s\right)\right\}\right)^{2}
$$

where $a v$ denotes average and $\hat{N}_{t u}^{*}(r, s)$ is the $(t, u)$ element of $\hat{\mathbf{N}}^{*}(r, s)$. Choice of values for $B, S$ and $R$ were chosen so that, in simulations, the variability in the estimates of each of the three components of variance (see (5)) were negligible.

The frequentist perspective views $\mathbf{N}$ as a fixed population total and $y$ as a fixed quantity. If all records on files $X$ and $Y$ are linked, then, from a frequentist perspective, $V_{t u}^{(y)}=\mathbf{0}$, and the only sources of variation in $\hat{\mathbf{N}}^{*}$ are due to incorrect linkage and due to estimating the linkage model, $\mathbf{Q}$.

\subsection{Linking Files of Different Sizes}

Consider the general case where $X$ has $N$ records, $Y$ has $M$ records and there are $O$ linked records. There are also no duplicated records on either $X$ or $Y$. Previously we considered 1-1 linkage, that is, $O=N=M$. Here we consider the two other important cases, $O<N=M$ and $O=N<M$.

\subsubsection{Case 1: $L<O=M$}

Linking only a subset of records is common in practice because a cut-off, $c_{o}$, for linked pair weights is usually enforced. Without loss of generality, we assume that the first $O$ records in $X$ are linked. The estimator of $\mathbf{Q}$ developed in Subsection 2.4 is no longer appropriate, since it is based on the assumption that all records on $X$ are linked. Here we are interested in estimating the $O \times N$ matrix $\tilde{\mathbf{Q}}$ with $i$ th row $\tilde{\mathbf{Q}}_{i}=\left(\tilde{q}_{i j}\right)$, where $\tilde{q}_{i j}$ is the probability that record $i$ in $X, i=1, \ldots, O$ is linked to record $j$ in $Y, j=1, \ldots, N$. Some suggested methods of estimating $\tilde{\mathbf{Q}}$ are given below. 
1. Purist approach. This involves estimating $\mathbf{Q}$ as described in Subsection 2.4, but where the $b$ th replicate is only kept if the set of $X$ records linked in the $b$ th replicate is the same as the set of $X$ records that were originally linked. This could be computationally infeasible if many replicates are discarded.

2. Bayes' Rule. This first involves estimating $\mathbf{Q}$ as described in Subsection 2.4. Then, conditioning on the set of $L$ linked records in $X$ and using Bayes' Rule, we get $\tilde{q}_{i j}=q_{i j}\left(\sum_{j=1}^{N} q_{i j}\right)^{-1}$.

3. Exchangeability. Here we assume an exchangeable structure (see (1)) for the linkage model, conditional on the set of $L$ linked records in $X$. Accordingly, it follows that $\tilde{q}_{i i}=\lambda=L^{-1} \sum_{i=1}^{L} q_{i j}\left(\sum_{j} q_{i j}\right)^{-1}$ and $\tilde{q}_{i j}=(1-\lambda)(N-1)^{-1}$ for $i \neq j$, where $\lambda$ is the average probability of a correct link.

For $\hat{N}_{t u}^{*}$ to remain unbiased when we replace $\mathbf{Q}$ with $\tilde{\boldsymbol{Q}}$ in (1), the conditional distribution of $y$ given $x$ for the $O$ linked records must be the same as for all $N$ records in $X$. That is, nonlinkage is completely at random. It is possible to relax this assumption to some degree and to improve the accuracy of $\hat{N}_{t u}^{*}$ by assuming that the conditional distributions of $y$ given $(x, z)$ for the linked records and for all records on $X$ are the same. Here $z$ is an auxiliary categorical variable defined on $X$ with categories $1 \leq v \leq V$. This is equivalent to assuming that nonlinkage is at random given $z$. Let $N_{t u v}$ and $N_{t u v}^{*}$ denote the true and linked counts defined by $x \times y \times z$ cross tabulation. Estimating $N_{t u v}$ given $N_{t u v}^{*}$ is straightforward, since we can simply treat $(x, z)$ as a more detailed version of $x$. Let $\hat{\mathbf{N}}^{*}=\left[\hat{N}_{t u v}^{*}\right]$ denote the bias-corrected estimates (4) defined by this more detailed cross tabulation. Our estimate of $N_{t u}$ then is $\hat{N}_{t u}^{*}=\sum_{v} \hat{N}_{t u v}^{*}$. The more general case, where the nonlinkage is not at random (i.e., there is no available $z$ that can be used to make the linked and unlinked distributions of $y, x$ and $z$ the same), requires further research.

\subsubsection{Case 2: $O=N<M$}

In this case there are more $y$ records than $x$ records, and all $x$ records are linked. Here $\mathbf{Q}=\left[\mathbf{Q}_{m}, \mathbf{Q}_{\bar{m}}\right]$ is $N \times M$, where $\mathbf{Q}_{m}$ is the $N \times N$ linkage model for records on $Y$ with a match, $\mathbf{Q}_{\bar{m}}$ is the $N \times(M-N)$ linkage model for records in $Y$ without a match, and $\mathbf{I}^{(Y)}$ is $M \times Y$. Also, $E\left(\mathbf{I}^{(Y)} \mid \mathbf{I}^{(X)}\right)$ is undefined for records on $Y$ that do not have a corresponding record on $X$. This means that we cannot evaluate the expectation of the naive estimator, $\mathbf{N}^{*}$, and hence correct for its bias. To remedy this, let $E\left(\boldsymbol{I}^{(Y)} \mid \boldsymbol{I}^{(X)}\right)=\tilde{\Delta}=\left(\tilde{\Delta}_{m}^{T}, \tilde{\Delta}_{\bar{m}}^{T}\right)^{T}$, where $\tilde{\Delta}_{m}$ and $\tilde{\Delta}_{\bar{m}}$ are the model expectations for the records with and without a match, respectively. That is, the $(j, u)$ element of $\tilde{\Delta}$ is

$$
\tilde{\Delta}_{j u}= \begin{cases}\pi_{u \mid t} & \text { if } x_{j}=t \text { and } j \leq N \\ \tilde{\mu}_{u} & \text { if } j>N \\ 0 & \text { otherwise. }\end{cases}
$$

where $\tilde{\mu}_{u}$ is the mean value of $I_{i u}^{(Y)}$ for the $M-N$ records on $Y$ without a match. From (6) it follows that

$$
E\left(\mathbf{N}^{*} \mid \mathbf{I}^{(X)}\right)=\left(\mathbf{I}^{(X)}\right)^{T}\left(\mathbf{Q}_{m} \tilde{\Delta}_{m}+\mathbf{Q}_{\bar{m}} \tilde{\Delta}_{\bar{m}}\right)
$$


The bias of the naive estimator of $\mathbf{N}$ is therefore

$$
\begin{aligned}
E\left(\mathbf{N}^{*}-\mathbf{N} \mid \mathbf{I}^{(X)}\right) & =\left(\mathbf{I}^{(X)}\right)^{T}\left\{\left(\mathbf{Q}_{m} \tilde{\Delta}_{m}+\mathbf{Q}_{\bar{m}} \tilde{\Delta}_{\bar{m}}\right)-\left(\mathbf{I}_{N} \tilde{\Delta}_{m}\right)\right\} \\
& =\left(\mathbf{I}^{(X)}\right)^{T}\left\{\left(\mathbf{Q}_{m}-\mathbf{I}_{N}\right) \tilde{\Delta}_{m}+\mathbf{Q}_{\bar{m}} \tilde{\Delta}_{\bar{m}}\right\}
\end{aligned}
$$

which suggests the bias-corrected estimator (where a 'hat' denotes an estimate),

$$
\hat{\mathbf{N}}^{*}=\left(\mathbf{I}^{(X)}\right)^{T}\left(\mathbf{I}^{\left(Y^{*}\right)}-\left(\hat{\mathbf{Q}}_{m}-\mathbf{I}_{N}\right) \hat{\tilde{\Delta}}_{m}-\hat{\mathbf{Q}}_{\bar{m}} \hat{\tilde{\Delta}}_{\overline{\mathrm{m}}}\right) .
$$

Here $\hat{\mathbf{Q}}$ (and hence $\hat{\mathbf{Q}}_{m}$ and $\hat{\mathbf{Q}}_{\bar{m}}$ ) as well as $\hat{\tilde{\Delta}}_{m}$ can be calculated using the bootstrap methods outlined earlier. However, this leaves $\hat{\tilde{\Delta}}_{\bar{m}}$ to be evaluated in (7). It would be reasonable to assume that $\tilde{\Delta}_{\bar{m}}$ is known if $M$ is many times larger than $N$, in which case it could be estimated from all the records in $Y$ (since records without a match would make up the vast majority of records on this file). Alternatively, if $X$ can be assumed to be a random subsample from $Y$, then we may write $\hat{\tilde{\mu}}_{u}=\hat{\pi}_{t u}\left(\sum_{t^{\prime}} \hat{\pi}_{t^{\prime} u}\right)^{-1}$, which is the marginal mean of $I_{i u}^{(Y)}$.

Combining the above two cases leads to the general case $O<N<M$. Equation (7) can then be used in place of (4) in the bootstrap algorithm described earlier.

\subsection{Inference for Binary Variables}

Finally, we move away from the estimation of frequencies defined by cross tabulations of linked categorical variables to modelling the distribution of a binary variable. Logistic or log-linear models are commonly used with frequency tables (see Hosmer and Lemeshow 2000).

Define $\mathbf{Z}^{T}=\left[\mathbf{z}_{1}, \ldots, \mathbf{z}_{w}, \ldots, \mathbf{z}_{W}\right]$, where $\mathbf{z}_{w}$ is a binary vector of length $K$ commonly referred to as the $w$ th covariate pattern. Put $\mathbf{T}=\left(t_{1}, \ldots, t_{w}, \ldots, t_{W}\right)^{T}$ and $\mathbf{R}=\left(r_{1}, \ldots, r_{w}, \ldots, r_{W}\right)^{T}$, where $t_{w}$ and $r_{w}$ are the numbers of 'successful' and 'unsuccessful' cases for the $w$ th covariate pattern.

A model for the number of successful cases when population units are independently distributed is

$$
E\left(t_{w}\right)=m_{w} \mu_{w}
$$

where $\mu_{w}=g\left(\mathbf{z}_{w}^{T} \boldsymbol{\beta}\right), g()$ is the link function, and $m_{w}=t_{w}+r_{w}$ is the total number of cases. A standard estimate of $\boldsymbol{\beta}$, denoted by $\hat{\boldsymbol{\beta}}$, is obtained by solving the score equation

$$
\mathbf{H}=\mathbf{Z}^{T}(\mathbf{T}-\operatorname{diag}(\mathbf{M}) \boldsymbol{\mu})=\mathbf{0}
$$

where $\mathbf{M}=\mathbf{T}+\mathbf{R}, \mu=\left(\mu_{1}, \ldots, \mu_{w}, \ldots, \mu_{W}\right)^{T}$ and $\mu_{w}=\mu_{w}(\boldsymbol{\beta})$.

Now consider the case where, again due to linkage error, $\mathbf{T}$ and $\mathbf{R}$ are not available. We can define $\mathbf{N}$ (see Subsection 3.1) so that $\mathbf{N}=[\mathbf{T}, \mathbf{R}]$ is of dimension $W \times 2$. This is the situation discussed in Subsection 3.1 for the case where $y$ is binary and the categories of $x$ correspond to the set of covariate patterns. It follows that we can replace $\mathbf{T}$ and $\mathbf{R}$ in (8) by their estimates $\hat{\mathbf{N}}^{*}=\left[\hat{\mathbf{T}}^{*}, \hat{\mathbf{R}}^{*}\right]$, where $\hat{\mathbf{N}}^{*}$ is given by (4). Note that if the model covariates are all observed on $X$, then $\mathbf{M}=\mathbf{T}+\mathbf{R}$ and $\hat{\mathbf{M}}^{*}=\hat{\mathbf{T}}^{*}+\hat{\mathbf{R}}^{*}$ are the same. In general, however, this will not be the case. A biased-corrected estimator of $\boldsymbol{\beta}$, denoted by $\hat{\boldsymbol{\beta}}^{*}$, is 
therefore obtained by solving the adjusted score equation

$$
\mathbf{H}_{a d j}=\mathbf{Z}^{T}\left(\hat{\mathbf{T}}^{*}-\operatorname{diag}\left(\hat{\mathbf{M}}^{*}\right) \mu\right)=\mathbf{0} .
$$

It is easy to see that if $\mathbf{Q}$ is known, then (9) is an unbiased score equation. Using the same arguments as those underpinning (5), a large sample approximation to the covariance matrix of $\hat{\boldsymbol{\beta}}^{*}$ can be estimated by

$$
\hat{\mathbf{V}}\left(\hat{\beta}^{*}\right)=\hat{\mathbf{V}}^{(y)}+\hat{\mathbf{V}}^{*}+\hat{\mathbf{V}}^{(\hat{\psi})}
$$

Here $\hat{\mathbf{V}}^{(y)}=\left(\mathbf{Z}^{T} \hat{\mathbf{V}} \mathbf{Z}\right)^{-1}$, where $\hat{\mathbf{V}}$ is diagonal with $w$ th element $\hat{\mu}_{w}\left(1-\hat{\mu}_{w}\right)$, $\hat{\mu}_{w}=g\left(\mathbf{z}_{w}^{T} \hat{\beta}\right)$

$$
\hat{\mathbf{V}}^{*}=S^{-1} \sum_{s} B^{-1} \sum_{b}\left(\hat{\beta}^{*}(b, s)-\hat{\beta}^{*}(s) \hat{\beta}^{*}(b, s)-\hat{\beta}^{*}(s)\right)^{T},
$$

where $\hat{\beta}^{*}(b, s)$ and $\hat{\beta}^{*}(s)$ are the solutions to (9) when we replace $\hat{\mathbf{T}}^{*}$ by $\hat{\mathbf{T}}^{*}(b, s)$ and $\hat{\mathbf{T}}^{*}(s)$ respectively, and

$$
\hat{\mathbf{V}}^{(\hat{\psi})}=S^{-1} \sum_{s} R^{-1} \sum_{r}\left\{\hat{\beta}^{*}(r, s) \underset{1 \leq r^{\prime} \leq R}{\operatorname{av}}\left(\hat{\beta}^{*}\left(r^{\prime}, s\right)\right)\right\}\left\{\hat{\beta}^{*}(r, s) \underset{1 \leq r^{\prime} \leq R}{\operatorname{av}}\left(\hat{\beta}^{*}\left(r^{\prime}, s\right)\right)\right\}^{T}
$$

where $\hat{\beta}^{*}(r, s)$ is the solution to (9) when we replace $\hat{\mathbf{T}}^{*}$ by $\hat{\mathbf{T}}^{*}(r, s)$.

\section{Simulation Results}

\subsection{The Simulated Data}

We simulated data where files $X$ and $Y$ were each comprised of $N=1,000$ records. The variable $x$ was generated independently for each record such that $x=1$ with probability 0.75 and $x=2$ otherwise. The variable $y$ then takes the values 1 or 2 and are generated from the multinomial distribution with parameter $\pi=\left(\pi_{1 \mid 1}, \pi_{2 \mid 1}, \pi_{1 \mid 2}, \pi_{2 \mid 2}\right)$. We consider two possible values for $\boldsymbol{\pi}, \boldsymbol{\pi}^{(a)}=(0.7,0.05,0.05,0.2)$ and $\boldsymbol{\pi}^{(b)}=(0.6,0.1,0.2,0.1)$.

We consider the logistic model $\operatorname{Pr}\left(t_{l}=1 \mid \mathbf{z}_{l}\right)=1 /\left\{1+\exp \left(-\zeta_{l}\right)\right\}$ and $\zeta_{l}=z_{l}^{T}\left(\beta_{0}, \beta_{1}\right)$. Define $\delta()=$.1 if the argument is true and $\delta()=$.0 otherwise. We fit the model to the generated data $(y, x)$ above where the first covariate pattern is $\mathbf{z}_{1}=(1,1)$ when $x=1$ and $\mathbf{z}_{2}=(1,0)$ when $x=2$, and the number of successes and cases for the $k$ th covariate pattern is $t_{k}=\sum_{i} \delta\left(x_{i}=k, y_{i}=1\right), m_{k}=\sum_{i} \delta\left(x_{i}=k\right)$ respectively for $k=1,2$.

The 1,000 records in files $X$ and $Y$ were allocated to 100 blocks with ten records per block. There were five linking fields. In Scenario 1, the five linking fields had $C_{l}=5,5,4$, 4,4 categories for $l=1, . .5$, respectively. In Scenario 2 the five linking fields had $C_{l}=7$, $7,7,6,6$ categories. The value for each linking field in file $X$ was assigned independently and with equal probability from the set of possible categories.

In Scenario 1 the linking fields were assigned $M_{l}=0.8,0.6,0.6,0.6,0.6$. In Scenario 2, these assignments were $M_{l}=0.8,0.7,0.7,0.6,0.5$. The linking fields in file $Y, F_{l i}^{(Y)}$, were 
generated independently for each $i$ and $l$ according to:

$F_{l i}^{(Y)}=f_{l i}^{(X)}$ with probability $M_{l}$

$=$ a random and equal probability draw from the $\operatorname{set}\left\{\{1,2, \ldots\}-,\left\{f_{l i}^{(X)}\right\}\right\}$ otherwise.

This meant that $U_{l}=1 / C_{l}$.

\subsection{Linking Under 1-1 Constraint}

Records were linked under the 1-1 constraint. This involved:

1. Sort all record pairs by their weight from highest to lowest;

2. The first record pair in the ordered list is linked;

3. All record pairs containing either of the records linked in Step 2 are removed from the list;

4. Return to Step 2 until all records are linked.

Of note is that the proportion of correct links was 0.74 and 0.91 in Scenarios 1 and 2, respectively.

\subsection{Results}

The number of replicates used to estimate the linkage model was $B=300$. The proposed estimator of $\mathbf{Q}$ was unbiased in both scenarios (e.g., the average value of the diagonal was 0.74 in Scenario 1). In contrast, the LL estimator of $\mathbf{Q}$ was significantly biased in both scenarios - the average values of the corresponding diagonals were 0.5 and 0.62 in Scenarios 1 and 2, significantly different from the corresponding true values of 0.74 and 0.91 . The conclusion is that the LL method of estimating $\mathbf{Q}$ performs poorly under 1-1 linkage. Consequently, estimates of the regression coefficients on which they are based would be heavily biased with poor coverage.

In order to measure Coverage (for nominal 95\% confidence intervals), Bias (as a percentage of the corresponding true value) and Root Mean Squared Error (RMSE), the various approaches to linkage and analysis were applied to 300 independently generated versions of file $X$ and file $Y$ and $S=10$. When $\boldsymbol{\psi}$ was unknown, the variation in $\hat{\psi}$ was estimated with $R=10$. These results are summarised in Tables 1 and 2 . The main results are:

- Naive inference, which treats the linked file as if it was perfectly linked, can be significantly biased and has poor coverage properties.

- The proposed method has negligible bias and good coverage properties whether or not $\boldsymbol{\psi}$ (and hence $\mathbf{Q}$ ) was known.

- The accuracy of the proposed estimator is somewhat reduced when $\boldsymbol{\psi}$ is unknown. For example, in Table 1, Scenario 1 where $\boldsymbol{\pi}=\boldsymbol{\pi}^{(a)}$, the RMSE was 0.012 and 0.014 when $\psi$ was known and unknown, respectively.

- The magnitude of the bias in the naive estimator tends to be higher for regression coefficients compared with frequency counts, even though the underlying data are the same. For example, for Scenario 1 and $\boldsymbol{\pi}=\boldsymbol{\pi}^{(b)}$ the bias in the regression coefficients 


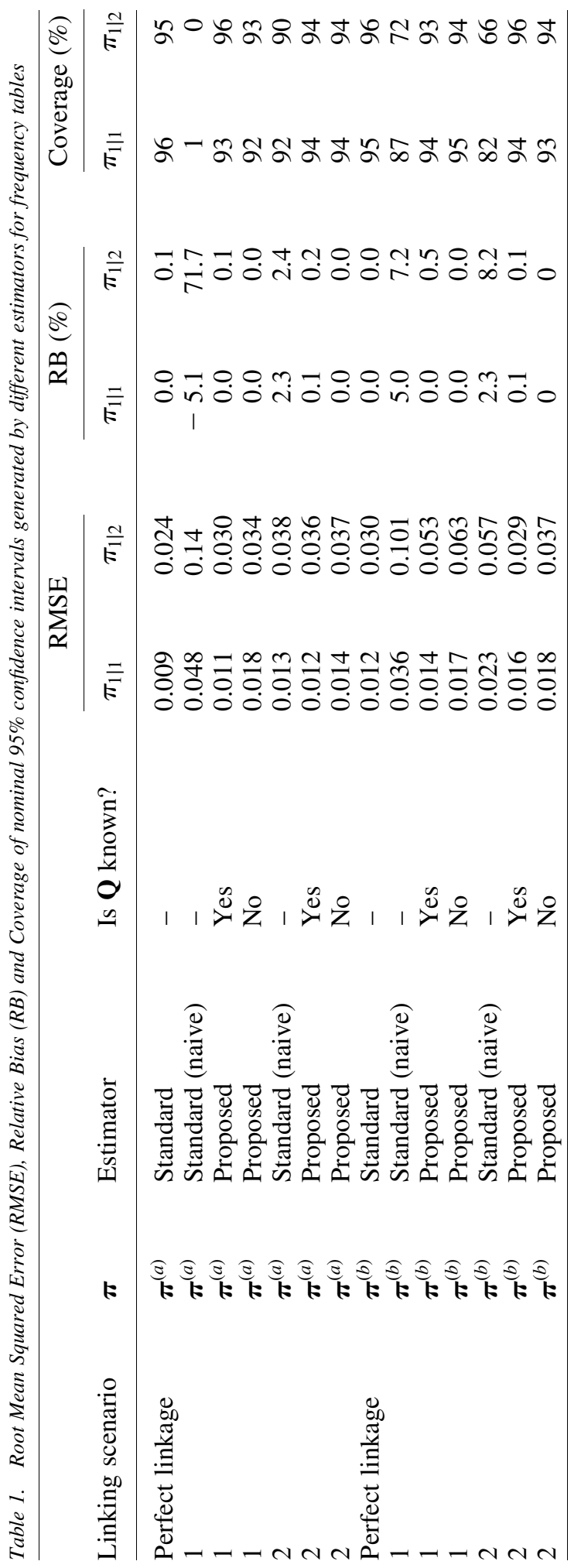




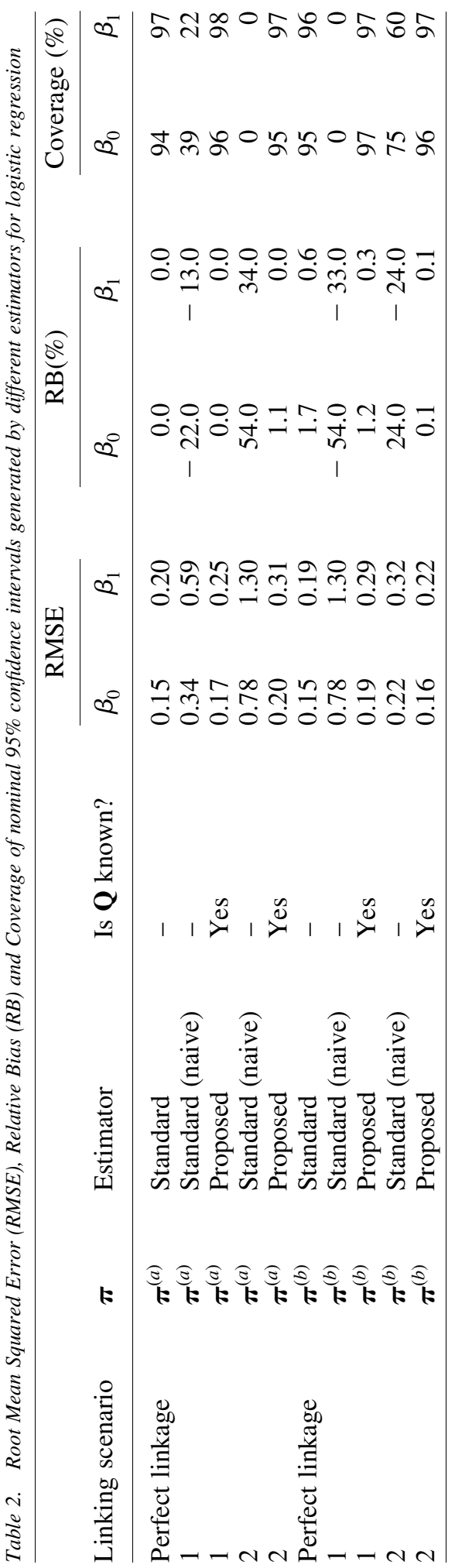


estimates is $54 \%$ and $-33 \%$, compared with $5 \%$ and $7 \%$ for frequency count estimates.

- The coverage rates for the naive estimator are sometimes close to the nominal $95 \%$ level for estimates of frequency counts, but are consistently lower than $95 \%$ for estimates of regression coefficients.

\section{Real Example}

The 2011 Census of Australian Population and Housing collected economic and social information from over 20 million people living in Australia with a reference date of 9 August 2011 (Census night). The Australian Department of Immigration and Citizenship's (DIAC) collected information about 1,315,000 people who were granted visas to live permanently in Australia from the beginning of 2006 to 9 August 2011; this information is stored on the Settlements Database (SD). Given that the undercoverage of the Census is small (less than 1\%) and all migrants in scope of the Census can be identified on the SD, it is reasonable to assume that the records on the SD are a subset of the records on the Census.

Two strategies were used to link the Census and SD (see Richter et al. 2013). The first linking strategy, called Bronze, did not use name and address. For the purpose of evaluation we focus on records linked during the fifth linking pass, which used the blocking variables date of birth and sex and linking variables country of birth, marital status, year of arrival in Australia, religion, and fine-level geography (see Richter et al. 2013). Probabilistic linking was performed using the 1-1 assignment algorithm in Febrl (Christen and Churches 2005). The second linking strategy, called Gold, used name and address and required significant evidence in order to assign a link (i.e., high cut off) such that we assume all Gold links are correct.

The true proportion of links made by Bronze linkage was $q=0.64$. That is, $64 \%$ of the Bronze links were also Gold links. Using the replication method in Subsection 2.4, $q$ was estimated to be $\hat{q}=0.65$. This is a remarkably accurate estimate and suggests that the Fellegi and Sunter (1969) framework, upon which the replication approach was based, is an accurate model for describing linkage errors.

Next we compare the estimator $\hat{N}^{* * *}$, using the Bronze links, to the corresponding population total N, calculated from Gold links. This comparison was made using only SD

Table 3. Cross tabulation of proportions according to level of qualification within Visa Class: Based on data obtained by linking settlements database to 2011 Census

\begin{tabular}{llccc}
\hline & & \multicolumn{3}{c}{ Level of qualification } \\
\cline { 3 - 5 } Visa class & Estimator & 1 & 2 & 3 \\
\hline 1 & True (gold) & 0.273 & 0.642 & 0.083 \\
& Naive (bronze) & 0.220 & 0.750 & 0.029 \\
2 & Proposed (bronze) & 0.220 & 0.755 & 0.025 \\
& True (gold) & 0.385 & 0.391 & 0.222 \\
& Naive (bronze) & 0.315 & 0.641 & 0.043 \\
& Proposed (bronze) & 0.343 & 0.551 & 0.105 \\
\hline
\end{tabular}


records that were linked by both Bronze and Gold - therefore any differences between the two are due only to incorrect links, the error of interest here. After restricting to 30-35year-olds on the SD there were about 3,000 records. As population estimates are of interest here, $y$ is a fixed quantity and $V_{t u}^{(y)}=\mathbf{0}$.

Table 3 sets out the true and naive frequency tables for level of qualification (Census) by visa class (SD). For simplicity, frequency counts are expressed as proportions of the marginal counts by visa class. Across the Visa Classes, the proposed estimates are closer (measured by the mean absolute difference) to the true proportions when compared with the naive estimates. However, for Visa Class 1 the naive estimates are marginally closer to the true proportions than the proposed estimates. Research into more robust ways of specifying and estimating the parameters in the linkage model is required.

\section{Conclusion}

Data linkage is being used increasingly by statistical organisations to link administrative data sets. This is because administrative data sets are rich sources of information and linking is a relatively inexpensive process. Probabilistic linking is an approach to linking data sets when there is no unique record key or identifier. This article proposes a method of inference using files that have been probabilistically linked. The method can accommodate 1-1 linking - in fact, as long as the linkage process can be replicated, the proposed method is valid. In this sense, there are good prospects of applying this method to linkage involving multiple passes. The proposed method worked well in a simulation study and showed promise in a real situation.

\section{References}

Chambers, R. 2009. "Regression Analysis of Probability-Linked Data." Statisphere Official Statistics Research Series 4. Available at: http://www.statisphere.govt.nz/ official-statistics-research/series/vol-4.htm (accessed August, 2013).

Chipperfield, J.O., G. Bishop, and P. Campbell. 2011. "Maximum Likelihood Estimation for Contingency Tables and Logistic Regression With Incorrectly Linked Data." Survey Methodology 37: 13-24.

Christen, P. and T. Churches. 2005. Febrl - Freely Extensible Biomedical Record Linkage. Available at: http://cs.anu.edu.au/ Peter.Christen/Febrl/febrl-0.3/febrldoc-0.3/ contents.html (accessed April 30, 2010).

Fellegi, I.P. and A.B. Sunter. 1969. "A Theory for Record Linkage." Journal of the American Statistical Association 64: 1183-1210. Doi: http://dx.doi.org/10.1080/ 01621459.1969 .10501049$.

Goldstein, H., K. Harron, and A Wade. 2012. "The Analysis of Record-Linked Data Using Multiple Imputation With Data Value Priors." Statistics in Medicine 31: 3481-3493. Doi: http://dx.doi.org/10.1002/sim.5508.

Herzog, T.N., F.J. Scheuren, and W.E. Winkler. 2007. Data Quality and Record Linkage Techniques. New York: Springer.

Hosmer, D.W. and S. Lemeshow. 2005. Applied Logistic Regression, (Second Edition). New York: John Wiley and Sons. 
Kim, G. and R. Chambers. 2012a. "Regression Analysis Under Probabilistic MultiLinkage." Statistica Neerlandica 66: 64-79. Doi: http://dx.doi.org/10.1111/j. 1467-9574.2011.00509.x.

Kim, G. and R. Chambers. 2012b. "Regression Analysis Under Incomplete Linkage." Computational Statistics and Data Analysis 56: 2756-2770.

Lahiri, P. 2003. "On the Impact of Bootstrap on Survey Sampling and Small Area Estimation.” Statistical Science 18: 199-210.

Lahiri, P. and M.D. Larsen. 2005. "Regression Analysis With Linked Data.” Journal of the American Statistical Association 100: 222-230. Doi: http://dx.doi.org/10.1198/ 016214504000001277.

Larsen, M.D. and D.B. Rubin. 2001. "Iterative Automated Record Linkage Using Mixture Models." Journal of the American Statistical Association 96: 32-41. Doi: http://dx.doi. org/10.1198/016214501750332956.

Richter, K., G. Saher, and P. Campbell. 2013. Assessing the Quality of Linking Migrant Settlement Records to 2011 Census Data. Methodology Research Papers, cat. no. 1351.0.55.043. Canberra: Australian Bureau of Statistics. Available at: http://www. ausstats.abs.gov.au/Ausstats/subscriber.nsf/0/D7A961FD8534DA6FCA257BC9001 17FBC/\$File/1351055043_aug\%202013.pdf (accessed December 1, 2013).

Scheuren, F. and W.E. Winkler. 1993. "Regression Analysis of Data Files that are Computer Matched." Survey Methodology 19: 39-58.

Winkler, W.E. 2001. Record Linkage Software and Methods for Merging Administrative Lists. Statistical Research Report Series, No. RR2001/03, Bureau of the Census. Available at: https://www.vrdc.cornell.edu/info7470/2007/Readings/rr2001-03.pdf (accessed April 30, 2010).

Winkler, W.E. 2005. Approximate String Comparator Search Strategies for Very Large Administrative Lists. Statistical Research Report Series, No. RRS2005/02. Bureau of the Census. Available at: https://www.census.gov/srd/papers/pdf/rrs2005-02.pdf (accessed April 30, 2010).

Winglee, M., R. Valliant, and F. Scheuren. 2005. "A Case Study in Record Linkage." Survey Methodology 31: 3-11.

Zhang, G. and P. Campbell. 2012. "Data Survey: Developing the Statistical Longitudinal Census Dataset and Identifying Its Potential Uses." Australian Economic Review 45: 125-133. Doi: http://dx.doi.org/10.1111/j.1467-8462.2011.00673.x.

Received January 2014

Revised November 2014

Accepted December 2014 